TRANSACTIONS OF THE

AMERICAN MATHEMATICAL SOCIETY

Volume 290, Number 2. August 1985

\title{
ON THE GROUP $\operatorname{SSF}(G)$, $G$ A CYCLIC GROUP OF PRIME ORDER ${ }^{1}$
}

BY

M. MALLER AND J. WHITEHEAD

\begin{abstract}
We extend the definition of the obstruction group $\operatorname{SSF}(G)$ in the case where $G$ is a cyclic group of prime order. We show that an endomorphism of a free $Z G$-module is a direct summand of a virtual permutation if its characteristic polynomial has the appropriate form. Among these endomorphisms the virtual permutations are detected by $K_{0}$. The main application is in detecting Morse-Smale isotopy classes.
\end{abstract}

1. Introduction. A diffeomorphism of a compact manifold $f: M \rightarrow M$ is called Morse-Smale if it satisfies Axiom A and strong transversality, and the nonwandering set $\Omega(f)$ is finite. From the point of view of dynamics these are the simplest structurally stable diffeomorphisms (see [9] for more details). In this paper we study the algebraic obstruction to the existence of a Morse-Smale diffeomorphism in a given isotopy class, in the case where the fundamental group $G=\pi_{1}(M)$ is a cyclic group of prime order.

Definition. A square integral matrix is called a virtual permutation (v.p.) if it has the block diagonal form

$$
\left(\begin{array}{lllll}
p_{1} & & & * & \\
& p_{2} & & & \\
& & \ddots & & \\
& 0 & & & p_{r}
\end{array}\right),
$$

where each block $p_{i}$ is either a signed permutation matrix or zero.

Theorem (Shub-Sullivan [9]). Suppose $\operatorname{dim} M \geqslant 6$ and $\pi_{1}(M)=0 . f: M \rightarrow M$ is isotopic to a Morse-Smale diffeomorphism if and only if $f$ can be represented on the integral chain level by virtual permutation matrices.

Franks and Shub [3] constructed an obstruction group, called SSF, which detects when this chain level condition can be satisfied. A linear map is called quasiunipotent if all eigenvalues are roots of unity, and quasi-idempotent if the zero

Received by the editors October 23, 1984.

1980 Mathematics Subject Classification. Primary 58F09; Secondary 20C10.

Key words and phrases. Morse-Smale diffeomorphism, virtual permutation, group rings.

${ }^{1}$ Research partially supported by PSC-CUNY Grant 1982-13945. 
eigenvalue is also allowed. By a change of basis the permutation blocks $p_{i}$ above can be put in the form

$$
\left(\begin{array}{ccccc}
0 & 1 & 0 & \ldots & 0 \\
0 & 0 & 1 & \ldots & 0 \\
\vdots & & & & \\
0 & 0 & \ldots & \ldots & 1 \\
\pm 1 & \ldots & \ldots & \ldots & 0
\end{array}\right)
$$

i.e. the companion matrix $C\left(x^{n}-1\right)$ of the polynomial $\left(x^{n}-1\right)$, so a Morse-Smale diffeomorphism must be quasi-unipotent on homology [8]. Let QI be the category whose objects are pairs $(M, e)$, where $M$ is a finitely generated $Z$-module and $e$ : $M \rightarrow M$ is quasi-idempotent on $M /$ Torsion $(M)$. Let $\mathbf{P} \subset \mathbf{Q I}$ be the full subcategory of objects which have finite resolutions by free v.p. endomorphisms. Then

$$
\mathrm{SSF}=K_{0}(\mathbf{Q I}) / K_{0}(\mathbf{P}) .
$$

If $E: C_{*} \rightarrow C_{*}$ is a quasi-idempotent chain map, let $\chi(E)=\sum_{i=0}^{\operatorname{dim}} C_{*}(-1)^{i}\left[E_{i}\right]$ in SSF.

Theorem (Franks-Shub [3]). An integral chain map E: $C_{*} \supset$ is chain homotopy equivalent to a v.p. chain map if and only if $E_{*}: H_{*}\left(C_{*}\right) \supset$ is quasi-idempotent and $\chi\left(E_{*}\right)=0$ in SSF.

In [4] Lenstra proved that SSF is nontrivial.

For nonsimply connected manifolds the appropriate algebraic models are the chain maps in the universal cover $C_{*}(M)$ over the group ring $Z G, G=\pi_{1}(M)$ [5]. V.p. matrices have the same block diagonal form but the blocks $p_{i}$ may have nonzero entries $\pm g, g \in G$; by a change of basis we can assume the $p_{i}$ are companion matrices of polynomials $x^{n} \pm g$.

Let $\operatorname{Mod}(G)$ be the category with objects $(M, e)$, where $M$ is a finitely generated $Z G$-module and $e: M \rightarrow M$ is an operator homomorphism associated with an automorphism of $G$. A morphism $h:(M, e) \rightarrow(N, f)$ in $\operatorname{Mod}(G)$ is a $Z G$-homomorphism $h: M \rightarrow N$ such that $f h=$ he. A resolution of $(M, e)$ in $\operatorname{Mod}(G)$ is a long exact sequence

$$
0 \rightarrow\left(N_{k}, f_{k}\right) \rightarrow \cdots \rightarrow\left(N_{0}, f_{0}\right) \rightarrow(M, e) \rightarrow 0 .
$$

Let $\mathbf{P} \subset \operatorname{Mod}(G)$ be the full subcategory of objects which have finite free v.p. resolutions. Let $\mathbf{Q} \supset \mathbf{P}$ be the full subcategory of $\operatorname{Mod}(G)$ of objects which are direct summands of objects of $\mathbf{P}$.

Define

$$
\operatorname{SSF}(G)=K_{0}(\mathbf{Q}) / K_{0}(\mathbf{P}) .
$$

In [6] we showed that if a $Z G$-chain map $E: C_{*} \rightarrow C_{*}$ has each $\left(C_{i}, E_{i}\right) \in \mathbf{Q}$, then $\left(C_{*}, E\right)$ is chain homotopy equivalent to a v.p. endomorphism if and only if

$$
\chi(E)=\sum_{i=0}^{\operatorname{dim} C_{*}}(-1)^{i}\left[E_{i}\right]=0
$$

in $\operatorname{SSF}(G)$. We were unable to directly identify the objects of $\mathbf{Q}$. 
When $G=0$ it follows from [3, Proposition 2.7] that $\mathbf{Q}=\mathbf{Q I}$. The proof depends on the fact that if $\xi$ is a primitive $k$ th root of unity, the ring $Z[\xi]$ is a Dedekind domain. In [7] we identified the objects of $\mathbf{Q}$ in a special case. By a $v$.p. polynomial in $Z G[x]$ we mean $x^{n}$ or a factor of $\left(x^{n} \pm g\right), g \in G$. When $G=Z^{n}, M$ is a free $Z G$-module and $e: M \rightarrow M$ is $Z G$-linear, we showed that $(M, e) \in \mathbf{Q}$ if and only if the characteristic polynomial of $e$ is a product of v.p. polynomials (equivalently the eigenvalues of $e$ are zero or roots of unity).

In this paper, using techniques of integral representation theory, we prove the following

THEOREM. Suppose $G$ is a cyclic group of prime order, $M$ is a free $Z G$-module and $E$ : $M \rightarrow M$ is $Z G$-linear. Then $(M, e) \in \mathbf{Q}$ if and only if the characteristic polynomial of $e$ is a product of $v$.p. polynomials.

This condition is clearly necessary. The rest of the paper is devoted to the proof of sufficiency. We would like to thank Michael Shub and John Franks for introducing us to this problem and for many helpful conversations.

2. Filtrations. A filtration of $(M, e)$ in $\operatorname{Mod}(G)$ is a sequence of submodules $0 \subset M_{1} \subset \cdots \subset M_{r}=M$ such that $e\left(M_{i}\right) \subset M_{i}$. By abuse of notation we will also denote as $e$ the quotient self-map of $M_{i} / M_{i-1}$.

LEMMA 1. If $(M, e)$ has a filtration with each $\left(M_{i} / M_{i-1}, e\right) \in \mathbf{Q}$, then $(M, e) \in \mathbf{Q}$.

Proof. Consider the exact sequence in $\operatorname{Mod}(G)$

$$
0 \rightarrow M_{1} \rightarrow M_{2} \rightarrow M_{2} / M_{1} \rightarrow 0 .
$$

By assumption, $M_{1}$ and $M_{2} / M_{1}$ have inverses $N$ and $N^{\prime}$ modulo $\mathbf{P}$. The sequence

$$
0 \rightarrow M_{1} \oplus N \rightarrow M_{2} \oplus N \oplus N^{\prime} \rightarrow M_{2} / M_{1} \oplus N^{\prime} \rightarrow 0
$$

is exact and the end terms belong to $\mathbf{P}$. But $\mathbf{P}$ is closed under short exact sequences [6, Lemma 2]. (One verifies that under our hypothesis the $M_{i}$ are projective.) Therefore $\left(M_{2}, e\right) \in \mathbf{Q}$ and the lemma follows by induction.

Henceforth we assume that $G$ is a cyclic group of prime order $p, G=\left\langle g \mid g^{p}=1\right\rangle$. We begin by constructing projective filtrations for endomorphisms of free $Z G$-modules.

The integral group ring $Z G \cong Z[x] / x^{p}-1$. Let $\phi(x)=1+x+\cdots+x^{p-1}$ so $x^{p}-1=(x-1) \phi(x)$, and let $I$ be the augmentation ideal of $Z G$, the kernel of $\omega$ : $Z G \rightarrow Z, \omega\left(\sum a_{i} g^{i}\right)=\sum a_{i}$. Then $I=\{\alpha \in Z G \mid \phi(g) \alpha=0\}=(g-1) Z G$ and $I \cong$ $Z[x] / \phi(x) \cong Z[\xi]$ as $Z G$-modules (where $g$ acts via $x$ or $\xi$ ), $\xi$ a primitive $p$ th root of unity.

The short exact sequence

$$
0 \rightarrow I \rightarrow Z G \stackrel{\omega}{\rightarrow} Z \rightarrow 0
$$

is a nonsplit extension of $Z[\xi]$ by $Z$, but over $Q$ the extension is split.

Let $Q G$ be the group algebra, $Q G \cong Q \otimes_{Z} Z G \cong Q[x] / x^{p}-1$. Let $J$ be the augmentation ideal of $Q G$; then $J \cong Q[\xi]=Q(\xi)$. Let $K=\{\alpha \in Q G \mid(g-1) \alpha=$ $0\}$; then $K \cong Q$, the trivial $Q G$-module. 
The sequence

$$
0 \rightarrow J \rightarrow Q G \stackrel{\omega}{\rightarrow} Q \rightarrow 0
$$

splits and so $Q G \cong Q(\xi) \oplus Q=J \oplus K$.

Let $M$ be a free $Z G$-module of rank $n, e: M \rightarrow M$ a $Z G$-linear homomorphism and suppose the characteristic polynomial of $e$ is $f_{1}(x) f_{2}(x) \cdots f_{r}(x), f_{i}(x) \in Z G[x]$ and degree $\left(f_{i}(x)\right)=s_{i}$. We construct a filtration of $M$ by first going up to $Q G$.

Let $S=\{m \in M \mid \phi(g) m=0\}$. If $\left\{m_{1}, \ldots, m_{n}\right\}$ is a basis for $M$ as a free $Z G$-module, an element $m=\sum \alpha_{i} m_{i} \in M$ belongs to $S$ if and only if each $\alpha_{i} \in I=$ $(g-1) Z G$. Thus $S=(g-1) M \cong Z[\xi]^{n}$.

Let $Q M=\left(Q \otimes_{Z} M\right) \supset 1 \otimes e$ and let

$$
Q S=Q \otimes_{Z} S=\{v \in Q M \mid \phi(g) v=0\} \cong Q(\xi)^{n} .
$$

Let $L=\{v \in Q M \mid(g-1) v=0\} \cong Q^{n}$. Then

$$
Q M \cong(Q G)^{n} \cong Q S \oplus L \cong Q(\xi)^{n} \oplus Q^{n}
$$

and both summands are invariant under $e$. Suppose $e$ is represented in the basis $\left\{m_{1}, \ldots, m_{n}\right\}$ by a $Z G$-matrix $\left(a_{i j}\right)$. Then $e: L \rightarrow L$ is represented by the $Q$-matrix $\left(\omega\left(a_{i j}\right)\right)$, where $\omega: Q G \rightarrow Q, g \rightarrow 1$, and $e: Q S \rightarrow Q S$ is represented by the $Q(\xi)$-matrix $\left(\theta\left(a_{i j}\right)\right)$, where $\theta: Q G \rightarrow Q(\xi), g \rightarrow \xi$.

Since $Q(\xi)$ and $Q$ are fields there exist $e$-invariant filtrations of $Q S$ and $L$ by subspaces

$$
0 \subset Q S_{1} \subset \cdots \subset Q S_{r}=Q S, \quad 0 \subset L_{1} \subset \cdots \subset L_{r}=L
$$

such that $e:\left[Q S_{i} / Q S_{i-1}\right] \supset$ has characteristic polynomial $\theta\left(f_{i}(x)\right) \in Q(\xi)[x]$ and $e$ : $\left(L_{i} / L_{i-1}\right) \unlhd$ has characteristic polynomial $\omega\left(f_{i}(x)\right) \in Q[x]$.

Let $(Q M)_{i}=Q S_{i} \oplus L_{i}$. Then $0 \subset Q M_{1} \subset \cdots \subset Q M_{r}=Q M$ is a filtration, $Q M_{i} / Q M_{i-1}$ is a free $Q G$-module of rank $s_{i}=$ degree $f_{i}(x)$ and $e:\left(Q M_{i} / Q M_{i-1}\right) \supset$ is annihilated by $f_{i}(x)$. Let $M_{i}=M \cap(Q M)_{i}$, so $(Q M)_{i}=Q \otimes M_{i}, 0 \subset M_{1} \subset \cdots$ $\subset M_{r}=M$ is a filtration of $M$ and $e:\left(M_{i} / M_{i-1}\right) \supset$ is annihilated by $f_{i}(x)$. If $q$ is any prime let $F_{q}=Z / q Z$, the finite field of $q$ elements. If $M$ is a $Z G$-module let $\bar{M}=M / q M$ be the reduced module over $F_{q} G$. Let $Z_{q}$ denote $Z$ localized at the prime ideal $q Z$ and let $M_{q}=Z_{q} \otimes_{Z} M$. Recall that a $Z G$-lattice is a $Z G$-module which is free and finitely generated as a $Z$-module [1, p. 245]. We will need the following fact:

LeMma 2. Let $A$ be a finite dimensional algebra over $F_{p}$ containing $F_{p} G$, and let $M$ be a ZG-lattice such that $\bar{M}$ is an $A$-module and $\bar{M} /(g-1) \bar{M} \cong(A)^{r} /(g-1)(A)^{r}$ as $A$-modules for some $r \geqslant 1$. If $\operatorname{rank}_{F_{p}} \bar{M}=\operatorname{rank}_{F_{p}}(A)^{r}$, then $\bar{M} \cong(A)^{r}$ as $A$-modules.

Proof. $\operatorname{rad}\left(F_{p} G\right)=(g-1) F_{p} G$ by $[1,5.24]$ and $(g-1) A \subset \operatorname{rad} A$. Therefore by $[1,5.1$ and 5.29]

$$
\frac{\operatorname{rad}(A)^{r}}{(g-1)(A)^{r}}=\operatorname{rad}\left[\frac{(A)^{r}}{(g-1)(A)^{r}}\right] \text { and } \frac{\operatorname{rad} \bar{M}}{(g-1) \bar{M}}=\operatorname{rad}\left[\frac{\bar{M}}{(g-1) \bar{M}}\right] \text {. }
$$

Thus $\bar{M} / \operatorname{rad} \bar{M} \cong(A)^{r} / \operatorname{rad}(A)^{r}$ as $(A / \operatorname{rad} A)$-modules. 
It follows that $(A)^{r}$ is the projective cover of $\bar{M}$ as an $A$-module $[1,6.23]$. Therefore there exists an epimorphism $\varepsilon:(A)^{r} \rightarrow \bar{M}$. Since $\operatorname{rank}_{F_{p}}(A)^{r}=\operatorname{rank}_{F_{p}} \bar{M}$, $\operatorname{ker}(\varepsilon)=0$ and $\varepsilon$ is an isomorphism.

LEMMA 3. $M_{i} / M_{i-1}$ is a projective $Z G$-module.

Proof. Observe first that $M_{i} / M_{i-1}$ is torsion-free over $Z$. Therefore $M_{i} / M_{i-1}$ is a $Z G$-lattice and

$$
\operatorname{rank}_{Z}\left(\frac{M_{i}}{M_{i-1}}\right)=\operatorname{rank}_{Q}\left(Q \otimes\left(\frac{M_{i}}{M_{i-1}}\right)\right)=\operatorname{rank}_{Q}\left(\frac{Q M_{i}}{Q M_{i-1}}\right)=s_{i} p,
$$

where $s_{i}=\operatorname{degree}\left(f_{i}(x)\right)$. Let $S_{i}=M \cap Q S_{i}=M_{i} \cap S=\left\{m \in M_{i} \mid \phi(g) m=0\right\}$. Then $S_{i}$ is a free $Z$-module of rank $s_{i}(p-1)$.

By $[1,31.3$ and 30.11$]$ it is enough to show that $\overline{\left[M_{i} / M_{i-1}\right]}=\bar{M}_{i} / \bar{M}_{i-1}$ is projective over $F_{p} G$. We show that $\bar{M} / \bar{M}_{r-1}$ is projective and the lemma follows by a finite induction.

Now $F_{p} G$ is a local ring and so by $[1,5.24$ and 5.19]

$$
\begin{aligned}
\operatorname{rad} \frac{\bar{M}}{\bar{M}_{r-1}} & =\operatorname{rad}\left(F_{p} G\right) \frac{\bar{M}}{\bar{M}_{r-1}}=(g-1) \frac{\bar{M}}{\bar{M}_{r-1}} \\
& =\frac{(g-1) \bar{M}+\bar{M}_{r-1}}{\bar{M}_{r-1}}=\frac{\bar{S}+\bar{M}_{r-1}}{\bar{M}_{r-1}} .
\end{aligned}
$$

Since

$$
\frac{S+M_{r-1}}{M_{r-1}} \cong \frac{S}{M_{r-1} \cap S}=\frac{S}{S_{r-1}}
$$

is a free $Z$-module of rank $s_{r}(p-1)$ we obtain $\left(\bar{S}+\bar{M}_{r-1}\right) / \bar{M}_{r-1}$ is an $F_{p}$-vector space of dimension $s_{r}(p-1)$. Similarly $\bar{M} / \bar{M}_{r-1}$ is an $F_{p}$-vector space of dimension $s_{r} p$. Therefore $\left(\bar{M} / \bar{M}_{r-1}\right) / \operatorname{rad}\left(\bar{M} / \bar{M}_{r-1}\right)$ is an $F_{p}$-vector space of dimension $s_{r}$. Let $B$ be a free $F_{p} G$-module of rank $s_{r}$. Then

$$
\frac{B}{\operatorname{rad} B} \cong \frac{\bar{M} / \bar{M}_{r-1}}{\operatorname{rad}\left(\bar{M} / \bar{M}_{r-1}\right)}
$$

as $\left(F_{p} G / \operatorname{rad}\left(F_{p} G\right)\right)$-modules and by Lemma $2, \bar{M} / \bar{M}_{r-1} \cong B$ as $F_{p} G$-modules. Hence $M / M_{r-1}$ is projective as a $Z G$-module. Therefore $M_{r-1}$ is projective, and $S_{r-1}=$ $M_{r-1} \cap S=(g-1) M_{r-1}$ and the induction continues.

Next we show that as in the case $G=0$, going up to $Q G$ the obstruction vanishes.

Lemma 4. Suppose $f_{i}(x)=x^{s} \pm g, g \in G$. Then $\left(Q M_{i} / Q M_{i-1}\right) \supset$ e is represented by a v.p. matrix over $Q G$.

Proof. We have

$$
\frac{Q M_{i}}{Q M_{i-1}}=\left(\frac{Q S_{i}}{Q S_{i-1}}\right) \oplus \frac{L_{i}}{L_{i-1}} \cong Q(\xi)^{s} \oplus Q^{s}
$$

with each term invariant under $e$, and $e: Q(\xi)^{s} \leftrightharpoons$ has characteristic polynomial $\theta\left(f_{i}(x)\right)=\left(x^{s} \pm \xi\right), e: Q^{s} \supset$ has characteristic polynomial $x^{s} \pm 1$. Let $t \in Z$ be 
respectively $p s, 2 p s, s, 2 s$ if $f_{i}(x)$ is $\left(x^{s}-g\right),\left(x^{s}+g\right)$, where $g \neq 1,\left(x^{s}-1\right)$, $\left(x^{s}+1\right)$. Let $H=\left\langle h \mid h^{t}=1\right\rangle$. Then we may regard $Q S_{i} / Q S_{i-1}$ as a module over the ring

$$
\frac{Q(\xi)[x]}{\left(x^{t}-1\right)} \cong Q(\xi) H
$$

and $L_{i} / L_{i-1}$ as a module over the ring

$$
\frac{Q[x]}{\left(x^{t}-1\right)} \cong Q H,
$$

where multiplication by $x$ is given in each case by $e$.

Let $N$ be a free $Z G$-module of rank $s$ and $e^{\prime}: N \rightarrow N$ an endomorphism represented by the companion matrix of $f_{i}(x)$; i.e. v.p. matrix. Then $Q N$ has a corresponding decomposition $Q N \cong Q S^{\prime} \oplus L^{\prime}$, where $Q S^{\prime}$ and $L^{\prime}$ are modules over $Q(\xi) H$ and $Q H$.

For each element $a \in Q(\xi) H$ consider the actions $\hat{a}:\left[Q S_{i} / Q S_{i-1}\right] \supset$ and $\hat{a}^{\prime}:$ $\left(Q S^{\prime}\right) \supset$. We claim the two actions have the same characteristic polynomials as $Q(\xi)$-homomorphisms. If $a \in Q(\xi)$ this is obvious, while if $h$ occurs in $a$ it follows since $\operatorname{char}(e)=\operatorname{char}\left(e^{\prime}\right)=x^{s} \pm \xi$. Similarly, for $a \in Q H$ the actions on $L_{i} / L_{i-1}$ and $L^{\prime}$ have the same characteristic polynomials as $Q$-homomorphisms.

Now by Maschke's theorem [1, 3.14] $Q(\xi) H$ and $Q H$ are semisimple rings. Therefore by a theorem of Brauer (see [10, p. 9, proof of Theorem 1.8]) modules over these rings are determined up to isomorphism by the characteristic polynomials of the actions of ring elements. Thus $\left(Q S_{i} / Q S_{i-1}\right) \cong Q S^{\prime}$ as $Q(\xi) H$-modules and $\left(L_{i} / L_{i-1}\right) \cong L^{\prime}$ as $Q H$-modules. Therefore

$$
\frac{Q M_{i}}{Q M_{i-1}}=\frac{Q S_{i}}{Q S_{i-1}} \oplus \frac{L_{i}}{L_{i-1}} \cong Q S^{\prime} \oplus L^{\prime} \cong N
$$

as $\left(Q G[x] / x^{t}-1\right)$-modules. Hence $e:\left[Q M_{i} / Q M_{i-1}\right] \supset$ is represented over $Q G$ by the v.p. matrix of $e^{\prime}$.

3. Proof of theorem. We restate our main result.

TheOREM. Let $G$ be a cyclic group of prime order $p, M$ a free $Z G$-module and $e$ : $M \rightarrow M Z G$-linear. $(M, e) \in \mathbf{Q}$ if and only if the characteristic polynomial of $e$ is a product of $v$.p. polynomials.

Proof. The condition is clearly necessary, we prove sufficiency. We have the characteristic polynomial of $e$ equals $f_{1}(x) f_{2}(x) \cdots f_{r}(x)$, where each $f_{i}(x) \mid x^{n_{i}} \pm h_{i}$, $h_{i} \in G$. By Lemma 1 it suffices to prove that each factor module $\left(M_{i} / M_{i-1}, e\right) \in \mathbf{Q}$. Observe that $x^{n} \pm h \mid x^{m}-1$, where $m=2 n p$. Let $N$ be a free $Z G$-module and $e^{\prime}$ : $N \rightarrow N$ an endomorphism such that $f_{i}(x) \operatorname{ch}\left(e^{\prime}\right)=x^{m}-1$.

Let $T=M_{i} / M_{i-1} \oplus N$ and $k=e \oplus e^{\prime}: T \rightarrow T$. By Lemma $3 T$ is a projective $Z G$-module and by Lemma $4 Q T$ is a free $Q G$-module with $\operatorname{ch}(k)=x^{m}-1$ on $Q T$. We prove that $(T, k) \in \mathbf{Q}$ through a series of lemmas, which will complete the proof of the theorem. 
Definition. Let $R$ be a Dedekind domain with quotient field $K$, and $A$ a finite-dimensional $K$-algebra. A commutative $R_{-}$order in $A$ is a commutative subring $\Lambda \subset A$ such that $\Lambda$ is finitely generated as an $R$-module and $K \Lambda=A[1,23.2]$.

Lemma 5. Let $\Lambda$ be a commutative $Z_{q}$-order in a finite dimensional $Q$-algebra $A$. For any pair of projective $\Lambda$-lattices $C$ and $D, Q C \cong Q D \Leftrightarrow C \cong D$ as $\Lambda$-modules.

Proof. By $[1,32.5]$ it is sufficient to show that $\bar{\Lambda} / \operatorname{rad} \bar{\Lambda}$ is a separable $F_{q}$-algebra (where reduction is $\bmod q$ ).

By $[1,30.3] \Lambda / \operatorname{rad} \Lambda \cong \bar{\Lambda} / \operatorname{rad} \bar{\Lambda}$ is a semisimple artinian ring. Since $F_{q}$ is perfect, every finite extension field $E$ of $F_{q}$ is separable over $F_{q}$. Let $E \otimes \bar{\Lambda}=\bar{\Lambda}^{E}$. Then $\operatorname{rad}\left(\bar{\Lambda}^{E}\right)=(\operatorname{rad} \bar{\Lambda})^{E}$ by $[1,7.9(\mathrm{i})]$ and

$$
\left(\frac{\bar{\Lambda}}{\operatorname{rad} \bar{\Lambda}}\right)^{E}=\frac{\bar{\Lambda}^{E}}{(\operatorname{rad} \bar{\Lambda})^{E}}=\frac{\bar{\Lambda}^{E}}{\operatorname{rad} \bar{\Lambda}^{E}}
$$

is a semisimple artinian ring. Hence $(\bar{\Lambda} / \operatorname{rad} \bar{\Lambda})^{E}$ is semisimple for each finite extension field $E$ over $F_{q}$. Therefore $\bar{\Lambda} / \operatorname{rad} \bar{\Lambda}$ is a separable $F_{q}$-algebra by [1, 7.3].

COROLlaRY. For all primes $q, T_{q}$ is a free $Z_{q} G$-module.

Proof. $T$ is a projective $Z G$-module and $Q T$ is a free $Q G$-module. The result follows since $Z_{q} G$ is a $Z_{q}$-order in $Q G$.

Definition. Let $R$ be a Dedekind domain with quotient field $K$ and let $\Lambda$ be an $R$-order in a separable finite-dimensional $K$-algebra $A$. Two $\Lambda$-lattices $C$ and $D$ are in the same genus $(C \vee D)$ if $C_{p} \cong D_{p}$ as $\Lambda_{p}$-modules for all maximal ideals $P$ of $R$ $[1$, p. 642].

Lemma 6. Let $\Phi(x) \in Z[x]$ be a cyclotomic polynomial and let $C$ be a projective $Z G$-module, $e: C \rightarrow C$ with $C$ annihilated by $\Phi(e)$. Let $D$ be a free ZG-module, $f$ : $D \rightarrow D$ an endomorphism represented by the companion matrix $C(\Phi(x))$. If $Q C \cong Q D$ as $(Q G[x] / \phi(x))$-modules, then $C \vee D$ as $(Z G[x] / \Phi(x))$-modules.

Proof. We must show that $C_{q} \cong D_{q}$ for all primes $q$.

Case 1. $q=p . C_{p} /(g-1) C_{p}$ is a torsion-free $\left(Z_{p}[x] / \Phi(x)\right)$-module. Since $Z_{p}[x] / \Phi(x) \cong Z_{p}[\eta], \eta$ a primitive $r$ th root of unity, it follows that $Z_{p}[x] / \Phi(x)$ is a Dedekind domain and hence $C_{p} /(g-1) C_{p}$ is projective as a $\left(Z_{p}[x] / \Phi(x)\right)$-module. By hypothesis $Q C \cong Q D$. As before

$$
Q C \cong \frac{Q[x]}{\Phi(x)} \oplus \frac{Q(\xi)[x]}{\Phi(x)} \text { and } \quad \frac{Q C}{(g-1) Q C} \cong \frac{Q[x]}{\Phi(x)} .
$$

It follows that $Q\left(C_{p} /(g-1) C_{p}\right) \cong Q\left[Z_{p}[x] / \Phi(x)\right]$ and by Lemma $5 C_{p} /(g-1) C_{p}$ $\cong Z_{p}[x] / \Phi(x)$. Hence

$$
\frac{\bar{C}}{(g-1) \bar{C}} \cong \frac{F_{p}[x]}{\Phi(x)} \cong \frac{A}{(g-1) A}
$$


a $A$-modules where $A=F_{p} G[x] / \Phi(x)$. By Lemma $2 A \cong \bar{C}$ as $A$-modules. Hence $C_{p}$ is a projective $\left(Z_{p} G[x] / \Phi(x)\right)$-module $[1,30.11]$ and by Lemma $5 C_{p} \cong D_{p}$.

Case 2. $q \neq p$. Since $\Phi(x)$ is a factor of $x^{s}-1$ for some $s$, we may view $C_{q}$ as a $Z_{q} \tilde{G}$-module, where

$$
\tilde{G}=\left\langle x, y \mid x^{s}=1, y^{p}=1,[x, y]=1\right\rangle
$$

and identifying $G$ with $\langle y\rangle$.

Let $H=\langle x\rangle$. Then $[\tilde{G}: H]=p$ which is invertible in $Z_{q}$. Hence $C_{q}$ is a direct summand of $Z_{q} \tilde{G} \otimes_{Z_{q} H} C_{q H}$, where $C_{q H}$ denotes $C_{q}$ viewed as an $H$-module $[1,33.5]$. As above $C_{q H}$ is a torsion-free $\left(Z_{q}[x] / \Phi(x)\right)$-module and hence is projective. Since

$$
Z_{q} \tilde{G} \otimes_{Z_{q} H} \frac{Z_{q}[x]}{\Phi(x)} \cong \frac{Z_{q} G[x]}{\Phi(x)}
$$

as $\left(Z_{q} G[x] / \Phi(x)\right)$-modules it follows that $C_{q}$ is projective as a $\left(Z_{q} G[x] / \Phi(x)\right)$-module. Lemma 5 then implies that $C_{q} \cong D_{q}$.

Lemma 7. Let $(C, e)$ and $(D, f)$ be as in Lemma 6. If $C \vee D$ as $(Z G[x] / \Phi(x))$ modules, then $(C, e) \in \mathbf{Q}$.

Proof. We first show that $(D, f) \in \mathbf{P}$. There exist polynomials $f_{1}(x) f_{2}(x) \cdots$ $f_{t}(x)$ and $h(x)$ such that

$$
f_{1}(x) \cdots f_{t}(x) \Phi(x)=h(x), \quad f_{i}(x)=x^{s_{i}}-1,
$$

and $h(x)=x^{n}-1$. By [2] $C(h(x))$ is similar over $Z$ to

$$
Y=\left(\begin{array}{cccc}
C\left(f_{1}(x)\right) & & & * \\
& \ddots & & \\
0 & & C\left(f_{t}(x)\right) & \\
& & & C(\Phi(x))
\end{array}\right)
$$

If $N_{1}$ and $N_{2}$ are free $Z G$-modules with endomorphisms $k_{1}$ and $k_{2}$ represented by $Y$ and $C(h(x))$ respectively, then $N_{1} \cong N_{2}$ as $(Z G[x] / h(x))$-modules, i.e. $\left(N_{1}, k_{1}\right) \in$ P. Let $N_{3}$ be the free $Z G$-module with endomorphism $k_{3}$ represented by

$$
\left(\begin{array}{ccc}
C\left(f_{1}(x)\right) & & * \\
0 & \ddots & \\
& & C\left(f_{t}(x)\right)
\end{array}\right) .
$$

There exists a short exact sequence of $(Z G[x] / h(x))$-modules

$$
0 \rightarrow N_{3} \rightarrow N_{1} \rightarrow D \rightarrow 0 \text {. }
$$

Since $N_{3}, N_{1} \in \mathbf{P}$ we obtain $D \in \mathbf{P}$ since $\mathbf{P}$ is closed under short exact sequences [6]. But $C \vee D$, hence there exists a $(Z G[x] / \Phi(x))$-module $L$ such that $L \oplus C \cong D \oplus D$ $[1,31.7]$, i.e. $(C, e) \in \mathbf{Q}$ as required. 
LEMMA 8. Let $T$ be a projective ZG-lattice with $k: T \rightarrow T$ a ZG-homomorphism. Suppose $Q T$ is a free $Q G$-module with $\operatorname{ch}(k \otimes 1)=x^{m}-1$ on $Q T$. Then $(T, k) \in \mathbf{Q}$.

Proof. $Q T$ is a free $Q G$-module by $[1,32.11]$. Factor $x^{m}-1$ into a product of irreducible factors in $Q[x]$. Then there exists a filtration

$$
0 \subseteq U_{1} \subseteq U_{2} \subseteq \cdots \subseteq U_{r-1} \subseteq U_{r}=Q T
$$

such that $U_{i} / U_{i-1}$ is a free $Q G$-module with $k \otimes 1$ having characteristic polynomial $\Phi_{i}(x)$ on $U_{i} / U_{i-1}, \Phi_{i}(x)$ cyclotomic. Then $k \otimes 1$ may be represented by $C\left(\Phi_{i}(x)\right)$ on $U_{i} / U_{i-1}$.

Let $T_{i}=T \cap U_{i}$. By Lemma $3 T_{i} / T_{i-1}$ is a projective $Z G$-lattice. By Lemmas 6 and $7\left(T_{i} / T_{i-1}, k\right) \in \mathbf{Q}$ and so by $\operatorname{Lemma} 1(T, k) \in \mathbf{Q}$.

This completes the proof of the theorem.

\section{REFERENCES}

1. C. W. Curtis and I. Reiner, Methods of representation theory with applications to finite groups and orders, Vol. I, Wiley, New York, 1981.

2. J. Franks and C. Narasimhan, The periodic behavior of Morse-Smale diffeomorphisms, Invent. Math. 48 (1978), 279-292.

3. J. Franks and M. Shub, The existence of Morse-Smale diffeomorphisms, Topology 20 (1981), 273-290.

4. H. W. Lenstra, Jr, Grothendieck groups of Abelian group rings, J. Pure Appl. Algebra 20 (1981), $173-193$.

5. M. Maller, Fitted diffeomorphisms of non-simply connected manifolds, Topology 19 (1980), 395-410.

6. Algebraic problems arising from Morse-Smale dynamical systems, Geometric Dynamics (Proc. Rio de Janeiro, 1981., Ed., J. Pallis, Jr.), Lecture Notes in Math., vol. 1007, Springer-Verlag, Berlin, 1983, pp. 512-521.

7. M. Maller and J. Whitehead, Virtual permutations of $Z\left[Z^{n}\right]$-complexes, Proc. Amer. Math. Soc. 90 (1984), 162-166.

8. M. Shub, Morse-Smale diffeomorphisms are unipotent on homology (Proc. Sympos. Dynamical Systems, Salvador, 1971), Academic Press, New York, 1973.

9. M. Shub and D. Sullivan, Homologv theory and dynamical systems, Topology 14 (1975), 109-132.

10. R. G. Swan and E. G. Evans, K-theory of finite groups and orders, Lecture Notes in Math., vol. 149, Springer-Verlag, Berlin, 1970.

Department of Mathematics, Queens College (CUNY), Flushing, New York 11367

Department of Computer Science, Queens College (CUNY), Flushing, New York 11367 